\title{
Comparison of early postoperative quality of life in minimally invasive versus conventional valve surgery
}

\author{
Tatsuya Yamada ${ }^{1}$, Ryoichi Ochiai ${ }^{1}$, Junzo Takeda ${ }^{1}$, Hankei Shin ${ }^{2}$, and Ryohei Yozu ${ }^{2}$ \\ ${ }^{1}$ Department of Anesthesiology, School of Medicine, Keio University, 35 Shinanomachi, Shinjuku-ku, Tokyo 160-8582, Japan \\ ${ }^{2}$ Department of Surgery, School of Medicine, Keio University, Tokyo, Japan
}

\begin{abstract}
Purpose. Minimally invasive cardiac surgery (MICS), an approach in which full sternotomy is avoided and the surgical incision is minimal, has been shown to produce less postoperative discomfort and to enable earlier mobilization and discharge than conventional cardiac surgery (CCS). This study was performed to retrospectively evaluate quality of life following MICS in comparison with CCS valve surgery.

Methods. Sixty-six patients scheduled for MICS and 50 patients scheduled for CCS for isolated aortic or mitral valve surgery from January 1999 to June 2001 were enrolled in the study. The clinical records for the two groups were compared across intraoperative parameters and those associated with postoperative quality of life.

Results. The aortic clamp and cardiopulmonary bypass times in the MICS group were longer than those in the CCS group $(144 \pm 42$ and $224 \pm 58 \mathrm{~min}$ vs $112 \pm 21$ and $179 \pm$ $27 \mathrm{~min}, P<0.001$ ). Postoperative pain medication (rectal buprenorphine and intramuscular pethidine) was administered to 18 of the 66 MICS patients $(27 \%)$, as compared with 26 of the $50 \mathrm{CCS}$ patients $(52 \%, P=0.007)$. Postoperative delirium was less frequent in the MICS group than the CCS group $(26 \%$ vs $44 \%, P=0.039)$. Initial postoperative food intake and urine catheter removal were possible earlier in the MICS than in the CCS group. MICS patients had shorter stays in the intensive care unit than CCS patients $(37.4 \pm 7.3$ vs $45.9 \pm 8.7 \mathrm{~h}, P<0.001)$.

Conclusion. Although longer aortic clamp and cardiopulmonary bypass times remain a problem in MICS procedures, our results suggest that MICS, as compared with CCS, facilitates earlier recovery of daily activities and provides improved quality of life in the early postoperative period.
\end{abstract}

Key words Minimally invasive cardiac surgery $\cdot$ Postoperative pain · Postoperative delirium - Quality of life

Address correspondence to: T. Yamada

Received: January 7, 2003 / Accepted: April 22, 2003

\section{Introduction}

Minimally invasive cardiac surgery (MICS) has been growing in popularity over the past several years, and incisions have become increasingly smaller as videoassisted endoscopic techniques have been developed. MICS has evolved two subcategories: beating heart myocardial revascularization without cardiopulmonary bypass, and valve surgery using femoral perfusion via a smaller incision, such as anterolateral minithoracotomy or various types of partial sternotomies. At our institution, MICS valve surgery was introduced in December 1996, while conventional valve surgery via a median sternotomy has also been traditionally used.

MICS valve surgery, an approach in which full sternotomy is avoided and the size of the surgical incision is minimal, has been shown to produce less postoperative discomfort and to enable earlier mobilization and discharge than conventional cardiac surgery (CCS) [1,2]. The term "minimally invasive" is derived from a reduction in surgical trauma and implies similar reductions in postoperative pain, impairment of usual activities, and length of hospital stay and rehabilitation. However, whether or not MICS procedures are really "minimally invasive" in this expanded sense remains unproven, as there is no general agreement on the definition and no standard approach to the measurement of outcomes covered by the term.

In cardiothoracic surgery, the measurement of outcome traditionally involves evaluation of cardiac symptoms, including mortality, complications, and recurrence of symptoms. Recently, increased attention has been given to postoperative quality of life, to provide a more comprehensive assessment of the impact of disease and treatments on the daily living activities of individual patients. Quality of life comprises selfperception of symptoms, well-being, and physical and mental function. In the present study, postoperative pain, incidence of postoperative delirium, and recovery 
of usual activities were evaluated as a means of assessing quality of life, and we retrospectively compared the quality of life in the early postoperative period following MICS and CCS valve surgery.

\section{Materials and methods}

We retrospectively reviewed the clinical records of patients scheduled for isolated aortic valve replacement or mitral valve replacement or repair from January 1999 to June 2001. Patients who needed reoperation and those who were tracheally extubated on and after the second postoperative day were excluded from the study. All valve operations were performed by teams of two staff surgeons using the MICS or CCS approach. The choice of the approach was based on the surgeon's preference and the patient's choice. This study was approved by the institutional ethical committee for human research.

MICS aortic valve replacement was performed using a 6- to 8-cm median skin incision and a "J" ministernotomy with upper semitransverse division at the level of the second rib with the aid of video-assisted endoscopic technique. The left femoral artery and the right femoral vein were cannulated to establish cardiopulmonary bypass. MICS mitral valve operations were performed using the ministernotomy technique described above, and cardiopulmonary bypass was established through cannulation of the left femoral artery, the right femoral vein, and the right internal jugular vein.

The CCS aortic valve replacement employed a full sternotomy and cannulation of the ascending aorta and the right atrium for cardiopulmonary bypass. CCS mitral valve operations were performed using the full sternotomy, and cardiopulmonary bypass was established through cannulation of the ascending aorta and the superior and inferior venae cavae.

Except for the cannulation, cardiopulmonary bypass and myocardial preservation were same in the MICS and CCS procedures. The ascending aorta was crossclamped and cardiopulmonary bypass was conducted at a moderately hypothermic temperature (bladder temperature, $28^{\circ} \mathrm{C}$ ) using a centrifugal pump, a membrane oxygenator, and an arterial line filter. Nonpulsatile pump flow was maintained at $2.2-2.61 \cdot \mathrm{min}^{-1} \cdot \mathrm{m}^{-2}$ with mean systemic pressure maintained between 50 and $80 \mathrm{mmHg}$. Myocardial preservation was performed by antegrade cold potassium blood cardioplegia in patients other than those with aortic valve stenosis, whereas patients with aortic valve stenosis received antegrade and retrograde cold potassium blood cardioplegia.

The anesthetic regimen for both the MICS and the CCS procedures consisted of induction with midazolam $(2-4 \mathrm{mg})$, fentanyl $\left(5 \mu \mathrm{g} \cdot \mathrm{kg}^{-1}\right), \quad$ and vecuronium $\left(0.15 \mathrm{mg} \cdot \mathrm{kg}^{-1}\right)$, followed by maintenance infusion of fentanyl $\left(50-75 \mu \mathrm{g} \cdot \mathrm{kg}^{-1}\right)$, midazolam $(0.2-$ $0.3 \mathrm{mg} \cdot \mathrm{kg}^{-1}$ ), and vecuronium. Intraoperative monitoring parameters included five-lead electrocardiography, arterial pressure, central venous pressure, pulmonary artery catheter, pulse oximetry, capnography, urine output, and nasopharyngeal and bladder temperature. Transesophageal echocardiography was also routinely used to assess ventricular and valvular function and to confirm the complete removal of intracardiac air.

Following completion of the surgery, patients were transferred to the intensive care unit. Postoperative care was standardized and was delivered by the same two staff anesthesiologists for all patients in the study. The patients were sedated with propofol (1$1.5 \mathrm{mg} \cdot \mathrm{kg}^{-1} \cdot \mathrm{h}^{-1}$ ) until the next morning and then were evaluated for tracheal extubation according to criteria including hemodynamic stability, adequate pulmonary function, adequate urine output, and minimal chest tube output. With tracheal extubation, the nasogastric tube was removed. About $6 \mathrm{~h}$ after extubation, the patients were allowed oral feeding of clear liquids if mentally alert. If the patient tolerated a clear liquid diet, a regular diet was provided on the next postoperative day. When a patient reported wound pain and required analgesics for it, rectal buprenorphine $0.4 \mathrm{mg}$ was administered in the intensive care unit and in the ward. If the patient was not satisfied within $30 \mathrm{~min}$, pethidine $35 \mathrm{mg}$ was given intramuscularly. Chest tubes were removed when the drainage volume decreased to less than $40 \mathrm{ml}$ in the previous $8 \mathrm{~h}$ and an air leak was not evident. The urinary catheter was removed after removal of the chest tubes and when the patient was able to ambulate around the bed and void without the catheter.

The clinical records were reviewed and the following variables were noted: aortic cross-clamp and cardiopulmonary bypass times, time to extubation, duration of postoperative inotropic support, time to first postoperative intake of food (regular diet), removal of chest tubes and urinary catheters, and intensive care unit and total hospital lengths of stay. In addition, the number of patients who required postoperative pain medication and those who developed postoperative delirium were evaluated. A patient was classified as having had delirium if the data from the medical records met ICD-10 Classification of Mental and Behavioural Disorders criteria $[3,4]$. A psychiatrist confirmed the diagnosis of delirium. The results are expressed as means \pm SD. Statistical analysis was performed using the Student's unpaired $t$-test and the chi-square test. Differences were considered significant when $P<0.05$. 
Table 1. Demographic and intraoperative data (means \pm SD)

\begin{tabular}{lccr} 
Characteristic & $\begin{array}{c}\text { MICS group } \\
(n=66)\end{array}$ & $\begin{array}{c}\text { CCS group } \\
(n=50)\end{array}$ & $P$ value \\
\hline Age & $\begin{array}{c}57 \pm 13 \mathrm{yr} \\
16 / 50\end{array}$ & $\begin{array}{c}57 \pm 10 \mathrm{yr} \\
15 / 35\end{array}$ & 0.941 \\
Sex (female/male) & & & 0.488 \\
Operation performed & 33 & 26 & 0.901 \\
$\quad$ Aortic valve replacement & 12 & 10 & \\
$\quad$ Mitral valve replacement & 21 & 14 & \\
$\quad$ Mitral valve repair & 30 & 21 & 0.711 \\
Hypertension & 6 & 4 & 0.797 \\
Diabetes mellitus & 16 & 12 & 0.992 \\
NYHA class & 40 & 30 & \\
$\quad$ I & 10 & 8 & \\
$\quad$ II & $2.8 \pm 0.7$ & $2.8 \pm 0.8$ & 0.934 \\
$\quad$ III & $144 \pm 42$ & $112 \pm 21$ & $<0.001$ \\
Cardiac index (l-min ${ }^{-1} \cdot \mathrm{m}^{-2}$ ) & $224 \pm 58$ & $179 \pm 27$ & $<0.001$ \\
Aortic clamp time (min) & $420 \pm 97$ & $363 \pm 53$ & $<0.001$ \\
Duration of cardiopulmonary bypass (min) & $530 \pm 99$ & $466 \pm 54$ & $<0.001$ \\
Operation time (min) & & & \\
Anesthetic time (min) & & & \\
\hline
\end{tabular}

MICS, minimally invasive cardiac surgery; CCS, conventional cardiac surgery; NYHA, New York Heart Association

\section{Results}

During the study period, a total of 86 patients underwent elective MICS valve surgery and 68 had elective CCS valve surgery. Reoperations were performed for bleeding in six of the MICS and four of the CCS group, and these patients were excluded from the study. In the remaining 144 patients, the postoperative requirement for mechanical ventilation ranged from 1 to 25 days. One hundred sixteen of the 144 patients were extubated on the first postoperative day (66 MICS and 50 CCS patients) and were included in the study. An additional 10 were extubated on the second postoperative day (4 MICS and 6 CCS patients), and 18 remained on mechanical ventilation for more than two postoperative days (10 MICS and 8 CCS patients). Of the 66 MICS and 50 CCS patients, $32 \mathrm{MICS}$ and $26 \mathrm{CCS}$ patients underwent aortic valve replacement, 12 MICS and 10 CCS patients underwent mitral valve replacement, and 21 MICS and 14 CCS patients had mitral valve repair.

Table 1 shows patient demographics and data for intraoperative parameters. There were no significant differences between the two groups in age or sex distribution or in the number of patients with hypertension or diabetes mellitus. None had clinical evidence of cerebrovascular disease, and preoperatively all were neurologically normal. Preoperative evaluation for cardiac function as assessed by New York Heart Association (NYHA) class and cardiac index did not differ between the groups. However, the duration of aortic clamp and cardiopulmonary bypass and surgical and anesthetic times were significantly longer in the MICS group.
Table 2 shows the data for postoperative parameters. There were no significant differences in postoperative extubation time or duration of postoperative inotropic support between the groups. Pain medication was given to 18 of the 66 patients in the MICS group; 16 received only rectal buprenorphine and 2 needed both rectal buprenorphine and intramuscular pethidine. In contrast, 26 of the 50 CCS patients required pain medication: 22 received buprenorphine and 4 had both buprenorphine and pethidine. Pain medication was markedly $(P=0.007)$ reduced following MICS procedures. Similarly, there were significantly fewer reports of postoperative delirium in the MICS group than in the CCS group ( $26 \%$ vs $44 \% ; P=0.039)$. In the MICS and CCS groups, the onset of delirium was at $2.4 \pm 0.6$ and $2.2 \pm 0.7$ postoperative days, and the duration of delirium was $3.5 \pm 0.8$ and $3.2 \pm 0.6$ days, respectively, with no significant differences. Although the timing of chest tube removal was similar in both groups, initial postoperative food intake and urine catheter removal were possible earlier in MICS patients than in the CCS group. The patients in the MICS group had a significantly shorter stay in the intensive care unit following surgery than those in the CCS group; however, there was no significant difference in length of total hospital stay.

\section{Discussion}

In the present study of the quality of life following MICS and CCS valve surgery, MICS procedures were 
Table 2. Postoperative data (means \pm SD)

\begin{tabular}{lccr}
\hline Measurement & $\begin{array}{c}\text { MICS group } \\
(n=66)\end{array}$ & $\begin{array}{c}\text { CCS group } \\
(n=50)\end{array}$ & $P$ value \\
\hline Postoperative extubation time (h) & $16.8 \pm 2.6$ & $17.1 \pm 1.7$ & 0.463 \\
Postoperative inotropic support time (days) & $3.0 \pm 1.6$ & $3.3 \pm 1.4$ & 0.343 \\
Postoperative pain medication & $18 / 66(27 \%)$ & $26 / 50(52 \%)$ & 0.007 \\
Postoperative delirium & $17 / 66(26 \%)$ & $22 / 50(44 \%)$ & 0.039 \\
First food intake (POD) & $2.4 \pm 0.6$ & $2.9 \pm 0.6$ & $<0.001$ \\
Chest tube removal (POD) & $3.5 \pm 0.6$ & $3.6 \pm 0.9$ & 0.388 \\
Urinary catheter removal (POD) & $3.6 \pm 0.8$ & $4.5 \pm 1.1$ & $<0.001$ \\
Duration of intensive care stay (h) & $37.4 \pm 7.3$ & $45.9 \pm 8.7$ & $<0.001$ \\
Length of total hospital stay (days) & $18.9 \pm 6.2$ & $19.1 \pm 5.9$ & 0.839 \\
\hline
\end{tabular}

POD, postoperative day

associated with significantly less demand for pain medication, fewer reports of postoperative delirium, earlier initial postoperative food intake and urinary catheter removal, and shorter stays in the intensive care unit compared with CCS.

In our study, the cross-clamp time and duration of cardiopulmonary bypass and operation and anesthetic times were longer in the MICS than in the CCS group. Increased cardiopulmonary bypass time has been cited as a problem with MICS, as compared with CCS procedures $[5,6]$. The literature shows that the aortic clamp and bypass times and duration of surgery are longer in minimally invasive procedures, which is attributable to the deeper operative field and attendant technical difficulties [7-9]. Cohn et al. [10] reported a $40 \%$ increase in cardiopulmonary bypass time in minimally invasive as compared with conventional procedures. Lytle [5] noted in his editorial "Is a smaller incision worth a longer pump run?" That a longer cardiopulmonary bypass time can result in postoperative hemodynamic instability, such as low cardiac output syndrome. In this study, MICS patients, however, presented stable postoperative hemodynamics and did not differ from CCS patients in that regard.

Reduction of postoperative pain is a theoretical advantage of MICS, which is in agreement with the results from several reports [10-12]. Svensson and D'Agostino [12] found reduced requirements for morphine and oral narcotics in patients following MICS. However, other studies have reported no differences between MICS and CCS in postoperative pain and demand for pain medication [13,14]. Walther et al. [13] reported that there was no difference in demand for postoperative pain medication between MICS and CCS patients, and that patients having a lateral minithoracotomy experienced the most severe pain during the first two postoperative days. Many types of incisions have been used for MICS, including minithoracotomy, parasternal incision, transverse sternotomy, and several types of partial sternotomies. In our study, ministernotomy was used for MICS, and we found that MICS was associated with a reduced demand for pain medication compared with CCS. Cooley [6] stated in his review that the length of a sternotomy incision is not a factor in recovery, but the degree of retraction of the sternum has an impact on surgical invasiveness and postoperative pain: patients have less pain and recover more quickly if the sternum is not overstretched. CCS patients in our study underwent full sternotomy with wide retraction for thoracic cannulation for cardiopulmonary bypass as well as to facilitate a good operative field, which may have resulted in increased postoperative pain in these patients. In contrast, cardiopulmonary bypass for MICS patients was established by means of the femoral vessels with or without the internal jugular vein, and the surgical approach was performed with the aid of a video-assisted endoscopic technique to avoid wide retraction, which might have contributed to reduced postoperative pain medication in MICS patients.

Neurologic complications following cardiac surgery range from transient neuropsychological dysfunction to serious complications such as stroke. The likelihood of perioperative stroke varies between $1 \%$ and $5 \%$, whereas transient neuropsychological dysfunction such as subtle cognitive and intellectual impairment (e.g., postoperative delirium or confusional states) is far more common as an adverse neurological outcome. According to the ICD-10 Classification of Mental and Behavioral Disorders, the essential features of delirium include clouding of consciousness with reduced ability to focus, sustain, or shift attention; changes in cognition, such as memory deficit, disorientation, language disturbance, and perceptual disturbance; disturbance of the sleep-wake cycle; disturbance of the level of psychomotor activity; and relatively rapid onset and a course that typically fluctuates $[3,4]$. The occurrence of postoperative delirium can result in increased morbidity, delayed functional recovery, and a prolonged hospital stay. The reported incidence of postoperative delirium varies from $2 \%$ to $57 \%$ in patients undergoing cardiac surgery 
[4]. In the present study, postoperative delirium was observed in $26 \%$ patients in the MICS group and $44 \%$ in the CCS group. This difference was significant.

Preoperative and intraoperative risk factors for adverse neurological outcomes after cardiac surgery were found to be advanced age, diabetes mellitus, preexisting cerebrovascular diseases, intracardiac (valvular) procedures, and long cardiopulmonary bypass time. [15-17]. In our study, age, the number of patients with hypertension and diabetes mellitus, and the type of surgery were similar in both groups. No patient had clinical evidence of cerebrovascular disease preoperatively. In patients undergoing cardiac surgery, hypoperfusion during cardiopulmonary bypass and microemboli of air or intracardiac thrombi resulting in brain ischemia are two major factors implicated in the development of postoperative confusion [18,19]. As Cooley [6] points out, evacuation of air from the heart is more difficult with the MICS technique than with CCS, because the surgeon cannot lift the apex of the left ventricle to aspirate contained air. Transesophageal echocardiography was useful in the detection of intracardiac air and thrombus and may have contributed to a reduced incidence of embolization, especially in MICS patients.

Postoperative factors play an important role in the incidence of postoperative delirium: physical and psychological stress, such as pain and anxiety are incentive factors in the genesis of delirium [20]. Extended time on ventilator treatment and low cardiac output syndrome with high-dose inotropic support are also thought to be the predicting factors for delirium [20,21]. Stay in the intensive care unit is stressful to patients because of its particular environment, which includes a high sound level, the absence of normal day-night cycle, sleep deprivation, sensory deprivation, and the inability of intubated patients to talk. In this study, although there were no significant differences in postoperative extubation time or duration of postoperative inotropic support between the groups, MICS patients experienced reduced postoperative pain and had shorter stays in the intensive care unit following their operations than CCS patients. This might explain the lower incidence of postoperative delirium in the MICS group.

We compared the recovery of usual activities between the MICS and CCS groups. Initial postoperative food intake and urinary catheter removal were earlier in the MICS group than in the CCS group. On removal of the tubes and catheters, the patients were able to stand up and walk around the ward, representing the recovery of daily activities. Earlier food intake may also have been conducive to the patient's feeling of well-being. Our results indicate that MICS is associated with less patient morbidity, faster recovery of usual activities, and improved quality of life during the early postoperative hospitalization period.
A fast-track, or early extubation, cardiac surgery program can provide stable perioperative hemodynamics and adequately suppress the perioperative stress response [22]. The fast-track anesthetic technique is a balanced anesthesia with low-dose narcotics and can allow early extubation. However, the risks (e.g., cardiorespiratory instability and bleeding) sometimes outweigh the potential benefit of early extubation. In this study, we did not follow the fast-track program, and the anesthetic regimen was total intravenous anesthesia with intermediate- to high-dose narcotic. Patients were sedated until the next morning and were assessed for tracheal extubation after evaluation of hemodynamic and respiratory stability and postoperative bleeding. A fast-track program can facilitate early discharge from the intensive care unit and the hospital and can improve medical costs and resource use in cardiac surgery. In the present study, stay in the intensive care unit was significantly shorter in MICS patients than in CCS patients; however, the total length of hospital stay did not differ between the groups. For early discharge from both the intensive care unit and the hospital, preoperative patient education is important to reduce anxiety and to establish patients' expectations. We also need to make appropriate weaning and extubating guidelines, arrhythmia management, postvalvular anticoagulation protocols, and intensive care unit and hospital discharge criteria.

In conclusion, minimally invasive procedures were associated with decreased demand for pain medication, reduced incidence of postoperative delirium, earlier initial postoperative food intake and urinary catheter removal, and shorter stays in the intensive care unit. Although longer aortic clamp and cardiopulmonary bypass times remain a problem in MICS procedures, our results suggest that MICS, as compared with CCS, facilitates earlier recovery of daily activities and provides improved quality of life during the early postoperative hospitalization period.

\section{References}

1. Cosgrove DM 3rd, Sabik JF (1996) Minimally invasive approach for aortic valve operations. Ann Thorac Surg 62:596-597

2. Lin PJ, Chang CH, Chu JJ, Liu HP, Tsai FC, Chu PH, Chiang CW, Yang MW, Shyr MH, Tan PPC (1996) Video-assisted mitral valve operations. Ann Thorac Surg 61:1781-1787

3. The ICD-10 Classification of Mental and Behavioural Disorders: clinical descriptions and diagnostic guidelines. World Health Organization, Geneva, 1992

4. van der Mast RC, Roest FH (1996) Delirium after cardiac surgery: a critical review. J Psychosom Res 41:13-30

5. Lytle BW (1996) Minimally invasive cardiac surgery. J Thorac Cardiovasc Surg 111:554-555

6. Cooley DA (1998) Minimally invasive valve surgery versus the conventional approach. Ann Thorac Surg 66:1101-1105 
7. Chitwood WR Jr, Wixon CL, Elbeery JR, Moran JF, Chapman WH, Lust RM (1997) Video-assisted minimally invasive mitral valve surgery. J Thorac Cardiovasc Surg 114:773-782

8. Minale C, Reifschneider J, Schmitz E, Uckmann FR (1997) Single access for minimally invasive aortic valve replacement. Ann Thorac Surg 64:120-123

9. Fann JI, Pompili MF, Burdon TA, Stevens JH, St Goar FG, Reitz BA (1997) Minimally invasive mitral valve surgery. Semin Thorac Cardiovasc Surg 9:320-330

10. Cohn LH, Adams DH, Couper GS, Bichell DP, Rosborough DM, Sears SP, Aranki SF (1997) Minimally invasive cardiac valve surgery improves patient satisfaction while reducing costs of cardiac valve replacement and repair. Ann Surg 226:421-428

11. Mächler HE, Bergmann P, Anelli-Monti M, Dacar D, Rehak P, Knez I, Salaymeh L, Mahla E, Rigler B (1999) Minimally invasive versus conventional aortic valve operations: a prospective study in 120 patients. Ann Thorac Surg 67:1001-1005

12. Svensson LG, D'Agostino RS (1998) Minimal-access aortic and valvular operations including the " $\mathrm{J} / \mathrm{j}$ " incision. Ann Thorac Surg 66:431-435

13. Walther T, Falk V, Metz S, Diegeler A, Battellini R, Autschbach R, Mohr FW (1999) Pain and quality of life after minimally invasive versus conventional cardiac surgery. Ann Thorac Surg 67:1643-1647

14. Aris A, Cámara ML, Montiel J, Delgado LJ, Galán J, Litvan H (1999) Ministernotomy versus median sternotomy for aortic valve replacement: a prospective, randomized study. Ann Thorac Surg 67:1583-1588

15. Trehan N, Mishra M, Kasliwal RR, Mishra A (2000) Surgical strategies in patients at high risk for stroke undergoing coronary artery bypass grafting. Ann Thorac Surg 70:1037-1045
16. van Dijk D, Keizer AMA, Diephuis JC, Durand C, Vos LJ, Hijman R (2000) Neurocognitive dysfunction after coronary artery bypass surgery: a systematic review. $\mathbf{J}$ Thorac Cardiovasc Surg 120:632-639

17. Selnes OA, Goldsborough MA, Borowicz LM, Mckhann GM (1999) Neurobehavioural sequelae of cardiopulmonary bypass. Lancet 353:1601-1606

18. Shaw PJ, Bates D, Cartlidge NEF, French JM, Heaviside D, Julian DG, Shaw DA (1987) Neurologic and neuropsychological morbidity following major surgery: comparison of coronary artery bypass and peripheral vascular surgery. Stroke 18:700707

19. Moody DM, Brown WR, Challa VR, Stump DA, Reboussin DM, Legault C (1995) Brain microemboli associated with cardiopulmonary bypass: a histologic and magnetic resonance imaging study. Ann Thorac Surg 59:1304-1307

20. Parikh SS, Chung F (1995) Postoperative delirium in the elderly. Aneth Analg 80:1223-1232

21. Gokgoz L, Gunaydin S, Sinci V, Unlu M, Boratav C, Babacan A, Soncul H, Halit V, Inanir S, Ersoz A (1997) Psychiatric complications of cardiac surgery postoperative delirium syndrome. Scand Cardiovasc J 31:217-222

22. Cheng DC, Karski J, Peniston C, Asokumar B, Raveendran G, Carroll J, Nierenberg H, Roger S, Mickle D, Tong J, Zelovitsky J, David T, Sandler A (1996) Morbidity outcome in early versus conventional tracheal extubation after coronary artery bypass grafting: a prospective randomized controlled trial. J Thorac Cardiovasc Surg 112:755-764 